\title{
Chapitre 6 ONG environnementales arabes et gestion des aires protégées : des acteurs entre histoires nationales et paradigmes mondialisés
}

Géraldine Chatelard*

Introduction

Les années 1990 sont un tournant à plus d'un titre pour le milieu des organisations non-gouvernementales (ONG) environnementales dans les pays du Sud - entendus ici comme ceux qui sont les objets des interventions des organisations internationales de développement au sens large, incluant également le secteur de l'environnement. Les nouveaux mots d'ordre de bonne gouvernance et de délégation de pouvoir à la société civile émergent dans le champ discursif et programmatique du développement, tandis que les nouveaux paradigmes appliqués à la gestion des ressources naturelles renouvelables sont ceux de biodiversité et de développement durable. Ces deux types de discours se greffent l'un sur l'autre dans la Convention sur la diversité biologique (CDB) adoptée au Sommet de la Terre à Rio de Janeiro en 1992. Dans le cadre du soutien à la mise en œuvre de la CDB dans les pays du Sud, des lignes de financement et des modes d'intervention techniques et gestionnaires issus des champs jusque-là relativement autonomes du développement international et de la protection environnementale convergent ${ }^{1}$.

C'est également l'époque où les grandes organisations non-gouvernementales du Nord d'envergure internationale (ONGI), qui agissent dans des espaces de liberté de parole et d'opinion qui les rendent aptes à influer sur les agendas internationaux ${ }^{2}$, cherchent à mobiliser les organisations non-gouvernementales (ONG) des pays du Sud pour peser sur les agendas des gouvernements de leur pays d'origine. Cette mobilisation s'opère dans un double mouvement. D'une part, la plupart des ONGI offrent à certaines ONG des pays du Sud un statut consultatif ou d'associées, conférant à celles-ci une légitimité à intervenir sur la scène

\footnotetext{
Géraldine Chatelard est chercheurse à l'Institut français du Proche-Orient à Amman. FROGER G. et ANDRIAMAHEFAZAFY F., 2003. SMOUTS M.-C., 2001.
} 
internationale, ce qu'elles font notamment en marge ou au centre des grandes conférences internationales. D'autre part, les ONG des pays du Sud se prévalent de leur présence dans les arènes internationales pour étendre leur capacité à peser sur les politiques publiques de leurs pays d'origine soit en vue de contester l'action de l'État, soit pour se poser comme partenaires privilégiés de l'action publique ${ }^{3}$.

Les ONGI auxquelles on fera référence dans ce chapitre sont l'Union internationale pour la conservation de la nature $(\mathrm{UICN})^{4}$ et BirdLife International ${ }^{5}$. Dans le cadre de leur stratégie d'influence internationale, ces organisations ont créé des bureaux régionaux en divers points de la planète dont, pour la région arabe, à Amman au cours des années 2000. Cette implantation leur permet de fournir des ressources professionnelles et des emplois à tout un groupe de diplômés des pays arabes, mais également de patronner un petit nombre d'ONG environnementales de la région qui deviennent membres de l'UICN ou de BirdLife, processus qui favorise la participation des cadres de ces ONG arabes à des forums internationaux. C'est, pour ces cadres, l'occasion de se familiariser avec les nouveaux mots d'ordre du champ international de la protection environnementale, dont la biodiversité est un sous-champ, ou bien encore de se former aux méthodes du lobbying international, aux méthodologies de mise en œuvre de la CDB - dont les nouveaux modèles de partenariat entre les ONG et le secteur public pour la gestion de la biodiversité - et (utilisé juste après) aux stratégies d'accès aux financements internationaux.

Enfin, la décennie 1990 est marquée par la promotion du modèle néolibéral et de la Nouvelle Gestion Publique ${ }^{6}$. Ce nouveau paradigme gestionnaire, d'abord expérimenté par des réformes du secteur public dans plusieurs pays du Nord, a pour but de « chercher à introduire des marchés ou des quasi-marchés au sein des institutions de l'État-providence de manière à en renforcer l'efficience $»^{7}$. Le modèle déborde très rapidement de la sphère publique :

3 Chartier D. et Ollitrault S., 2006.

4 L'UICN, créée en 1948, se définit comme une association internationale de membres gouvernementaux et non gouvernementaux. Ces membres élaborent des normes en matière de protection de la biodiversité (dont les catégories d'aires protégées) qui servent de références internationales. Son histoire et ses modes d'action (elle aide les gouvernements à préparer des plans nationaux pour l'environnement et à identifier des projets auxquels elle apporte son appui technique) sont pleinement imbriqués avec ceux du développement durable. Elle est étroitement associée aux travaux du Programme des Nations Unies pour le Développement (PNUD) sur l'environnement et à ceux du Programme des Nations Unies pour l'environnement (PNUE) qui se réfèrent largement aux normes de l'UICN.

BirdLife International se définit comme une fédération mondiale d'organisations non-gouvernementales pour la protection des oiseaux. Elle compte aujourd'hui plus de cent membres actifs dits partenaires, en général un seul dans chaque pays, et des représentations régionales. Sa liste d'espèces aviaires en danger et son système de classification des Zones importantes pour la conservation des oiseaux font autorité auprès de l'UICN.

6 MERRIEN F.-X., 1999.

7 MERRIEN, F.-X, 1999, p. 95. 
plusieurs travaux de sciences sociales illustrent comment la pénétration des logiques du marché et des modèles gestionnaires dans les États du Nord ont amené à une diversification du champ associatif où coexistent à présent des organisations basées sur le bénévolat et le modèle non lucratif, mais également de nouveaux types d'associations qui se professionnalisent et combinent activités marchandes et non lucratives ${ }^{8}$. C'est généralement cette capacité à s'inscrire dans le paradigme néolibéral qui rend certaines associations séduisantes pour des États-providence en pleine transformation, qui cherchent à se décharger sur le secteur non-gouvernemental d'une partie de leurs prérogatives sociales ou économiques.

Dans le même temps, la Nouvelle Gestion Publique est préconisée aux gouvernements des pays du Sud par le Fonds monétaire international (FMI), la Banque mondiale et les autres organismes de crédit internationaux qui y voient le moyen de remédier à l'endettement public structurel. Malgré la diffusion du modèle dans les pays arabes, la recherche en sciences sociales tant occidentale qu'arabe n'a jusqu'ici guère porté attention aux effets de l'emprise grandissante du marché et des modèles gestionnaires sur les transformations de la sphère associative dans les pays du Moyen Orient et d'Afrique du Nord ${ }^{9}$. C'est en effet plutôt le rôle des ONG comme acteurs de la gouvernance et de la démocratisation qui a retenu l'attention ${ }^{10}$, ou bien encore la question du développement du marché et de sa relation avec les entrepreneurs et hommes d'affaires ${ }^{11}$.

Notre hypothèse est que, dans le champ des politiques de protection de la biodiversité tant à l'échelle supranationale ${ }^{12}$ que dans les contextes nationaux, l'adoption de ces nouveaux paradigmes et de nouvelles politiques d'échelles vont modifier les modalités de l'action publique et les configurations d'acteurs: organisations internationales ou inter-étatiques, ONGI, ONG des pays du Sud dont le cadre d'action a été jusque-là restreint à l'échelle nationale, et enfin acteurs des secteurs public et privé. Une autre transformation à l'œuvre est celle de la nature même de certaines ONG qui se projettent dans le champ supranational, s'ouvrent à la marchandisation et transforment leurs modes d'action et d'institutionnalisation dans le cadre de nouveaux types de relations avec les acteurs publics et privés.

Ce sont ces processus de transformation qui nous intéressent ici, illustrés à travers un

\footnotetext{
8 Biggs S. et NeAme A., 1995, SAlamon L. 1997, Ghinet S. et DunAND C., 1998, ainsi que ANHEIER H., 2005, p. 372.

9 On peut citer comme exceptions BRAND L., 2001 et BAYLOUNY A. M., 2010 pour la Jordanie, ainsi que ABU-SADA C., 2007 et Challand B., 2009 pour les Territoires palestiniens occupés.

10 Norton A. R., 1995 et 1996, et BEN NEFISSA S. et al. 2004.

11 Voir CAMAU M. (2002, p. 226) qui cite plusieurs travaux.

12 Nous entendons le concept de supranational comme concernant les échelles de relations entre États et organisations qui dépassent les souverainetés nationales.
} 
domaine particulier de l'action publique et associative, la gestion des aires protégées (AP), dans trois pays arabes du Moyen-Orient. La juxtaposition de trois cas d'études - au Liban, en Jordanie et en Syrie - vise à faire ressortir que l'introduction, dans trois systèmes de gouvernance très contrastés, de paradigmes développementaux et environnementaux similaires, de modèles gestionnaires présentés comme universalisables, et de dynamiques de mise en réseaux supranationale produit des effets différents en fonction des temporalités et contextes politiques nationaux. La suite de ce chapitre présente et analyse, en les restituant dans leur profondeur historique respective et dans les différents contextes nationaux d'institutionnalisation du champ public de la gestion des AP, la trajectoire et le processus de transformation de trois ONG environnementales arabes qui sont devenues des partenaires privilégiés des pouvoirs publics dans la gestion des $\mathrm{AP}^{13}$. La conclusion propose quelques pistes pour une comparaison.

Nous envisageons ces ONG comme inscrites dans un champ relationnel de contraintes et d'opportunités où elles cherchent à étendre leur capacité d'action et à améliorer leur position par rapport à d'autres acteurs aux objectifs similaires en mobilisant diverses formes de capital social $^{14}$. Au sein de ces associations, certains individus sont les acteurs principaux de ces dynamiques. Se pencher sur les acteurs permet de dépasser les dichotomies État/société civile et secteur privé/secteur associatif et de mieux comprendre comment, voire pourquoi, certains acteurs associatifs se sont trouvés en position d'interlocuteurs et de partenaires privilégiés à divers moments de l'histoire récente lorsque les politiques de protection de la biodiversité ont connu des réorientations. Cette approche permet en outre de rendre compte de la manière dont s'articulent les échelles supranationales et nationales dans le champ des politiques environnementales. En effet, les individus dont il sera question circulent entre plusieurs de ces sphères et échelles et utilisent diverses formes de capital acquises dans les unes pour améliorer leur position dans les autres. De fait, la fongibilité de différents types de capital social lors du passage d'une sphère ou d'une échelle à l'autre pose la question de l'autonomie de ces dernières.

\footnotetext{
13 Les données présentées dans ce chapitre ont été recueillies entre 1996 et 2010 dans plusieurs postures de recherche : un travail anthropologique sur le changement social et économique dans une AP du Sud jordanien (Chatelard G., 2003 et 2005), une étude socioéconomique dans trois aires protégées de Syrie (dont ChATElaRD G., 2008), des visites et entretiens dans deux aires protégées au Liban, la participation en tant qu'intervenante ou observatrice à plusieurs réunions et forums régionaux et internationaux d'ONG environnementales, et de nombreuses discussions formelles et informelles avec les personnes citées que je remercie de leur disponibilité. Le programme de l'ANR-Sud «Tanmia. Le développement : fabrique de l'action publique dans le monde arabe ? » m'a permis de donner une cohérence à ces données de terrain très disparates et de les problématiser en relation avec la question de l'action publique. 14

Au sens où l'entend Pierre Bourdieu (1980 et 1994).
} 


\section{La Société pour la protection de la nature au Liban}

Les AP, sous l'appellation de réserves naturelles (mahmiyat), existent depuis l'époque mandataire au Liban et relèvent de plusieurs ministères ou entités locales, en général les municipalités. Dans la période récente, elles ne constituent pas un secteur de grand intérêt public avant les années 1990 qui sont celles de l'après-guerre civil où l'État reconstitue les institutions publiques. Dans le contexte de la mise en œuvre par le Liban de la CDB, que le pays a ratifiée en 1994, la Banque mondiale incite à la création d'un Ministère libanais de l'Environnement (MdE) et à la constitution de nouvelles AP qui correspondent aux standards internationaux de l'UICN. Dans cette optique, la Global Environment Facility (GEF) ${ }^{15}$ octroie subventions et prêts au MdE. À la suite d'une mission exploratoire en 1994, l'UICN recommande la création de trois nouvelles AP (Arz al-Chouf, Palms Islands et Horsh Eddin). Selon Paul Kingston ${ }^{16}$, l'UICN est impressionnée par le dynamisme apparent des ONG environnementales au Liban face à la faible capacité gestionnaire des pouvoirs publics, y compris les municipalités dans l'après-guerre civile ${ }^{17}$. Aussi, sous l'influence des nouvelles approches promouvant la gestion participative, l'organisation recommande d'octroyer un rôle important aux ONG, et plus généralement à la société civile, dans la gestion des nouvelles $\mathrm{AP}^{18}$. Cette recommandation est intégrée dans le plan national de soutien à la création et gestion des AP qui a un double objectif: renforcer la capacité régulatrice de l'État et développer les compétences gestionnaires des municipalités pour la conservation des AP. Aux ONG nationales sont assignés deux rôles : l'intermédiation entre les municipalités et le MdE et la formation (sous le terme de renforcement des capacités) des membres des communautés

15 La GEF, créée en 1992, cogérée par la Banque mondiale, le PNUD et le PNUE, constitue une des principales sources de financement des programmes nationaux de protection de l'environnement liés à l'application de la CBD. Elle octroie en particulier des fonds d'amorçage pour la création d'AP soit aux gouvernements soit au secteur associatif, dans le dernier cas grâce à un programme de petites subventions (small grants).

KINGSTON P., 2001.

17 Sur les associations environnementales libanaises, voir la thèse de doctorat de Karam Karam (2004) qui préfère le terme d'associations écologistes. L'ouvrage du même auteur (2006) reprend la plus large partie de cette thèse mais est amputé des chapitres sur les associations écologistes.

$18 \quad$ Kingston déconstruit la catégorie de société civile au Liban à partir de l'exemple de la mise en place de la réserve du Chouf en territoire druze sous le contrôle de la famille Jumblat. Il montre comment les réseaux de patronage se sont assuré le contrôle de cette initiative via des ONG locales politiquement loyales, voire en créant des ONG ad hoc, et en se créant des monopoles environnementaux financés par l'extérieur. La gestion de la réserve du Chouf est restée aux mains du personnel des ONG avec une implication nominale des populations voisines : les représentants de la communauté locale sont sélectionnés sur la base de leur loyauté politique (Kingston P., 2001). Mes entretiens, menés en 2007 dans les réserves du Chouf et de Kfar Zabad (Békaa), témoignent que les liens de patronage sont toujours aussi importants. 
locales à la gestion environnementale. Pour chaque AP, le MdE constitue alors un comité qui rassemble l'ensemble de ces acteurs.

C'est dans ce cadre que les ONG peuvent recevoir des subventions, particulièrement de la GEF, pour mener à bien leur mission. Cependant, c'est à l'État qu'il revient de donner une légitimité à certaines ONG en tant que partenaires du MdE. Assad Sarhal, directeur d'une association libanaise dont on traitera plus loin, estime que «Les organisations internationales cherchent à renforcer le rôle du secteur public en matière de régulation et de coordination et, quand il s'agit d'octroyer des financements pour les AP, s'assurent que les associations ont l'aval du MdE ${ }^{19}$. Dans le contexte libanais, où l'État a été en recul marqué durant les années de guerre civile, les organismes financiers internationaux ne sont pas les seuls à encourager un renforcement du rôle des pouvoirs publics. Durant ce que Karam Karam nomme la « genèse du secteur écologique libanais » au cours des années d'après-guerre :

«D'une part, ce sont les associations écologistes qui contribuent à l'institutionnalisation du secteur de l'écologie en appelant les pouvoirs publics, à l'échelle nationale et locale, à assumer leurs responsabilités et à exercer leurs prérogatives. D'autre part, dans un contexte où priment décentralisation et privatisation, l'écologie devient un lieu de tension entre différents acteurs, locaux et centraux, mais surtout un moyen de générer plus d'État (et non moins d'État) ${ }^{20}$.

Pour autant, ce plus d'État ne signifie pas le tout État alors que le secteur public libanais se reconstitue précisément au moment où domine, chez les bailleurs internationaux, le paradigme de la Nouvelle Gestion Publique. Aussi, dès son institutionnalisation, le modèle de gestion des AP adopté au niveau national est configuré sur le modèle d'une délégation de l'action publique à des acteurs de la société civile.

Le milieu des organisations environnementales au Liban est très compétitif, dépassant plusieurs centaines d'associations anciennes et nouvelles ${ }^{21}$. Se pose la question du financement de leurs activités : il s'agit de se placer au mieux d'une part face aux bailleurs internationaux qui disposent de lignes de financement destinées aux organisations de la société civile, et d'autre part face à l'État qui redistribue une partie des subventions et prêts qui lui sont octroyés par les bailleurs. C'est là l'occasion de faire jouer le patronage politique. Le Forum libanais pour l'environnement (FLE), l'un des principaux rassemblements libanais d'ONG environnementales, dont les membres entretiennent des liens étroits avec certaines

\footnotetext{
$19 \quad$ Entretien avec A. Sarhal, 2008.

20 KARAM K., 2004, p. 339.

21 KARAM K., 2004, p. 341.
} 
personnalités politiques ${ }^{22}$, se donne pour objectif essentiel de faire pression sur le MdE pour obtenir que les mandats de gestion des AP, et les financements qui leurs sont associés, soient octroyés à ses membres ${ }^{23}$.

L'un des principaux acteurs associatifs qui émerge lors du processus d'extension de l'action publique au domaine des AP est la Société pour la Protection de la Nature au Liban (SPNL). Cette ONG, créée en 1986, concentre jusqu'à la fin des années 1990 ses activités sur l'éducation à l'environnement, la collecte des déchets et l'éducation à l'utilisation de l'eau, en produisant, entre autres, du matériel pédagogique pour le MdE. Une autre de ses activités est la protection des oiseaux. Dans les années 1990, la SPNL compte soixante membres et seulement deux salariés, avec un budget annuel modeste compris entre 20000 et 100000 euros répartis en moyenne entre $60 \%$ de subventions publiques, $10 \%$ de cotisations et $30 \%$ de dons. La SNPL est cependant déjà membre de plusieurs collectifs ou réseaux internationaux : elle est le partenaire associatif libanais de BirdLife, le premier membre libanais de l'UICN, est accréditée par le PNUE, etc. Ce sont ces statuts vis-à-vis d'organisations supranationales qui permettent à la SPNL d'être associée par le MdE à la réflexion sur la mise en place de la nouvelle génération $\mathrm{d}^{\prime} \mathrm{AP}^{24}$.

Assad Sarhal, le président de la SPNL, est diplômé en gestion environnementale d'une université américaine. Il participe à la création du FLE, dont il est élu deuxième président. C'est sans doute parce que sa position au sein du FLE lui permet plus qu'à d'autres de faire valoir ses compétences auprès du MdE qu'il est nommé administrateur de la réserve du Chouf bien qu'il ne soit ni originaire de la région ni druze ${ }^{25}$. Ce sera l'occasion pour lui de dépasser les savoirs théoriques acquis lors de sa formation universitaire et de sa fréquentation des ONGI en développant son expertise de gestionnaire de terrain. On peut lire dans son parcours les effets cumulatifs de plusieurs types de capital (éducatif, relationnel et expert) acquis ou développé à plusieurs échelles et qui lui permettent de faire carrière dans le contexte libanais et supranational en renforçant son profil d'expert gestionnaire.

Au début des années 2000, Sarhal commence à se poser en critique du mode de gestion mis en place dans le Chouf qu'il qualifie de conservationniste : les activités humaines (chasse, élevage, agriculture, collecte de bois et plantes, etc.), considérées comme prédatrices, sont interdites; la priorité absolue est donnée à la protection de la biodiversité ; et les

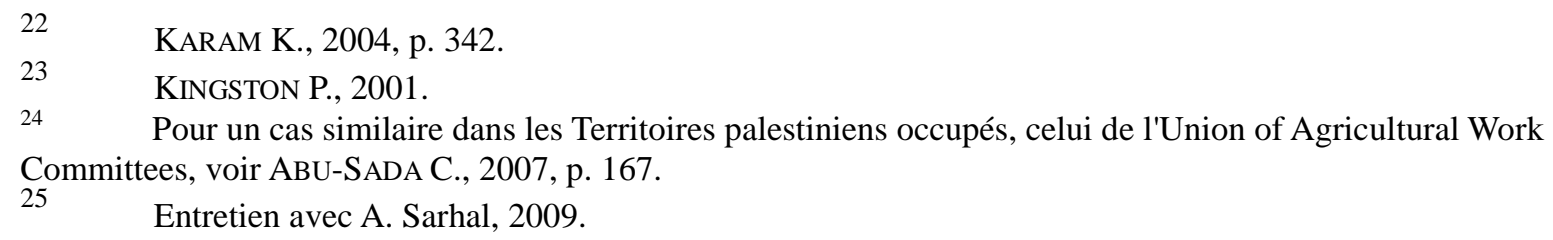


populations locales ne sont pas associées à la gestion. Sarhal considère que ce modèle a des effets pervers : les populations sont marginalisées et appauvries, elles ne s'approprient pas les objectifs de protection environnementale, et elles expriment leur opposition à l'existence de la réserve par le braconnage ou la collecte illégale de plantes hors de tout système traditionnel de régulation de l'usage des ressources naturelles ${ }^{26}$.

Sarhal donne alors une nouvelle impulsion à la SPNL, proposant un discours alternatif au modèle de gestion conservationniste. L'approche participative et socioéconomique qu'il prône dans le contexte libanais, et à laquelle il s'est familiarisé lors de sa formation universitaire et de ses interactions avec les ONGI, entre en écho avec les nouvelles orientations internationales en matière de gestion locale des AP. En effet, à partir de la fin des années 1990, sous l'impulsion de plusieurs ONGI (dont l'UICN et BirdLife) et de bailleurs internationaux (particulièrement la GEF et la Banque mondiale) une étape est franchie : l'idée d'associer à la gestion des ressources renouvelables dans les AP les ONG nationales et internationales mais aussi les communautés locales, le tout dans un souci de promouvoir la gouvernance locale et la conservation de la biodiversité ${ }^{27}$. Cette étape illustre un déplacement d'une participation/consultation vers une participation/négociation, dans la mesure où elle repose sur l'établissement de contrats visant à définir une cogestion locale entre l'État et les populations qui vivent dans ou à proximité des AP. En outre, à l'approche centrée sur la conservation s'est substituée celle du développement durable qui comprend un volet socioéconomique étroitement lié aux objectifs de protection environnementale. L'État, ou toute autre entité gestionnaire des AP, s'engage à développer des activités qui assurent aux populations locales un revenu supérieur à celui qu'elles tirent de l'exploitation des ressources naturelles de la réserve, le tout inscrit dans une stratégie de réduction de la pauvreté. Le volet socioéconomique des nouveaux modèles de gestion des AP fait de l'écotourisme la panacée capable de remplir ce rôle économique compensatoire. Il permet l'entrée dans des régions rurales, où l'économie est encore largement structurée autour d'activités de subsistance ou bien de dépendance aux ressources fournies par l'État, d'un modèle de développement par la croissance économique et l'accès des populations qualifiées de pauvres à un segment du

\footnotetext{
26

Entretien avec A. Sarhal, 2008.

27 Dans un document préparé à l'occasion du Cinquième Congrès mondial des Parcs (Durban, 2003), la Banque mondiale résume ainsi sa stratégie de financement au profit de nouveaux modèles de gestion des AP pour les années 1988-2003: «Through a combination of lending and grant assistance, the Bank is assisting client governments to seek creative ways to support appropriate models of protected area management by working with local, national and international NGOs, academic institutions, other donors and local community organizations. New projects are giving increasing emphasis to local community participation and their roles within protected area management ». (BANQUE MONDIALE, 2003a, p. 19).
} 
marché du tourisme, secteur économique structuré sur un mode capitaliste.

La SNPL approfondit une réflexion que Sarhal a déjà engagée depuis plusieurs années avec un petit groupe d'environnementalistes actifs dans les pays de la région, dont le Liban, l'Arabie Saoudite et des émirats du Golfe. Il s'agit de développer un nouveau concept de gestion des AP, la hima, adapté d'un système très ancien ayant existé dans la Péninsule Arabique et le Levant ${ }^{28}$. La hima modernisée a le quadruple objectif de protéger la biodiversité, de développer économiquement des communautés appauvries, de transmettre la gestion directe des AP aux populations locales, et d'ancrer ce modèle dans une tradition islamique pour le rendre culturellement légitime et acceptable par les populations locales ${ }^{29}$. Sarhal voit dans la hima le lien parfait entre la conservation des ressources renouvelables et le développement durable ${ }^{30}$.

Dans la seconde moitié des années 2000, Sarhal améliore sa position au sein des ONGI dont la SPNL est membre et se voit confier des mandats régionaux : il nommé conseiller international de Birdlife pour la région Moyen Orient et est élu vice-président du comité régional pour l'Asie occidentale de l'UICN. À ce titre, il est très présent dans les forums régionaux et internationaux. Fort des expériences locales que la SPNL a commencé à mettre en place dans trois $\mathrm{AP}^{31}$ au Liban, Sarhal fait mettre la hima sur l'agenda de plusieurs réunions régionales d'ONG et d'ateliers lors de forums internationaux au point qu'il gagne le soutien de BirdLife et de l'UICN à son initiative : les deux ONGI adoptent la hima comme un modèle régional à soutenir et pour lequel elles sont prêtes à octroyer des financements.

Retour à l'échelle libanaise, la SPNL se donne pour mission d'aider les municipalités, qui ont créé ou souhaitent créer une hima sur leur territoire, à planifier sa mise en œuvre et à trouver des financements internationaux. Une fois les fonds dégagés, la SPNL forme à la gestion environnementale un comité local dépendant de la municipalité, et fournit de

\footnotetext{
28 Selon Lutfallah Gari, un auteur saoudien adoptant une perspective historique et religieuse : «A himā is a reserved pasture, where trees and grazing lands are protected from indiscriminate harvest on a temporary or permanent basis. It existed in the Middle East before Islam; but it was treated as a private reserve for powerful chieftains who were said to have used it as a tool of oppression. With the emergence of Islam, its function changed; it became a property dedicated to the well-being of the whole community around it. Tribes had their own himās with the permission of the state, and acted as self-governing in the absence of state control. This institution flourished through the first half of the twentieth century, when major political, economical and social changes took place in the Levant and the Arabian Peninsula. » (GARI L., 2006, p. 213).

29 Pour une vision du modèle hima tel que préconisé par Sarhal et d'autres experts environnementaux de la région depuis le milieu des années 2000 voir UICN et SPNL, 2007.

30 Ce faisant, il fait l'impasse sur l'échec, bien documenté, de l'expérience de remise en vigueur de la hima tentée en Syrie dans les années 1980 pour améliorer la gestion des pâturages dans les steppes (BocCO R., 1993, $\mathrm{p}_{31}$ 349).

Ces AP sont : la hima de Kfar Zabad dans la Békaa, la hima de Ebel as-Saqi dans le Sud, et la hima marine de Qoleile dans le Nord.
} 
l'assistance technique en vue de la mise en place de projets générateurs de revenus, particulièrement l'écotourisme. La SPNL est aussi le garant institutionnel et scientifique de ces entreprises vis-à-vis des bailleurs et reçoit une partie des subventions pour son appui technique. L'implication de l'association dans la médiation et la mise en œuvre de ces projets locaux renforce la légitimité de la SPNL auprès du MdE qui cherche à améliorer les modes de gestion des AP au niveau national. En 2009, les activités de conseil de la SPNL auprès du MdE résultent en l'introduction, par décret ministériel, de la hima parmi les catégories d'AP au Liban $^{32}$. Pour Sarhal, qui agit au nom de la SPNL, il est essentiel de se faire reconnaître comme partenaire privilégié par les organismes internationaux et les bailleurs de fonds autant que par les pouvoirs publics libanais. En effet, ces derniers (si les pouvoirs pub alors ceux-ci), par leur action régulatrice, légitiment juridiquement le mode d'intervention participatif dont la SPNL est l'initiateur dans le cadre libanais.

Au niveau régional, une autre étape essentielle de l'institutionnalisation de la hima est franchie grâce à la création de fonds de financement dédiés. Là aussi, Sarhal a été le moteur de ces initiatives. Il a utilisé le réseau BirdLife et l'UICN pour convaincre le responsable de l'association environnementale qatarienne qui est partenaire de ces deux ONGI d'approcher la Sheikha Moza, épouse de l'Émir du Qatar, et présidente de la Qatar Foundation ${ }^{33}$. En 2009, grâce à une première subvention d'un million de dollars US octroyée par cette fondation, un fonds hima est créé au Qatar. Il a pour mission d'allouer une ou deux subventions par an à des ONG arabes membres de BirdLife pour mettre en place ou soutenir des projets de hima-s dans les Zones importantes pour la conservation des oiseaux, selon la classification utilisée par BirdLife. Fin 2010 un autre fonds hima était en voie de création en Arabie Saoudite, sous le patronage d'un membre de la famille royale, afin d'octroyer des subventions aux ONG arabes au-delà du réseau BirdLife. En se branchant sur le nouveau champ discursif de la protection environnementale islamique, que soutient l'UICN depuis les années 1990 et qui est en plein développement actuellement ${ }^{34}$, et en lui donnant une crédibilité opérationnelle grâce à la création de hima-s au Liban, Sarhal a stratégiquement utilisé le référent islamique pour diversifier les sources de financement des ONG environnementales arabes et mobiliser les monarchies du Golfe, qu'il a par ailleurs très consciemment mises en compétition ${ }^{35}$.

Ces échelles multiples, dans lesquelles Sarhal est personnellement impliqué en tant

Entretiens et correspondances avec A. Sarhal et des employés de la SPNL, 2008, 2009 et 2010

Cette fondation privée, créée en 1995 par l'Émir du Qatar et extrêmement bien dotée, est devenue en quelques années une source de financement très recherchée par le milieu associatif des pays arabes dans les domaines de l'éducation, de la culture, de la recherche scientifique et du développement.

$34 \quad$ Voir, par ex., UICN, 1994 et FoLTZ R. et ali, 2003

35 Entretien avec A. Sarhal, 2010. 
qu'expert mais également en tant que président de la SPNL, ont induit un développement de son association et une transformation de son activité qui fait actuellement figure de modèle en matière d'expertise gestionnaire. Karam ${ }^{36}$ fait état de transformations similaires affectant tout un pan du milieu des associations environnementales libanaises. Fin 2010, le budget de fonctionnement de la SPNL a considérablement augmenté, et la liste des bailleurs internationaux qui financent les projets dans lesquels l'association est impliquée est très longue. La part des organismes multilatéraux et bilatéraux dépasse de loin celle des subventions publiques. La SPNL compte sept salariés à plein temps et a dû repenser son organisation en profondeur en donnant la priorité à la professionnalisation. Ce tournant a été négocié en recrutant plus de personnel aux compétences techniques, en offrant à ceux déjà en poste des possibilités de formation à la gestion environnementale à l'international, et en s'appuyant moins qu'avant sur le volontariat ${ }^{37}$.

La SPNL se place aujourd'hui sur un champ porteur de l'action environnementale : celui de la gestion locale, ou cogestion, des AP. Elle est en concurrence avec d'autres ONG libanaises qui cherchent à mettre en place de petites AP dans leurs municipalités ou régions d'activités. Cependant, contrairement à la plupart de ces autres ONG dont la base de soutien et les actions sont très locales, Assad a su assurer à la SPNL des réseaux de soutien à plusieurs échelles: supranationale, nationale et municipale. Il a su non seulement obtenir des délégations de l'État libanais et l'institutionnalisation nationale du modèle de gestion des AP dont il a été l'initiateur, mais également sa reconnaissance internationale, qui semble avoir été une condition essentielle pour son adoption par le MdE. Il a également été capable de générer de nouvelles ressources financières régionales qui vont bénéficier à plusieurs acteurs associatifs arabes, dont les membres régionaux des ONGI qui ont parrainé la hima. Sarhal et la SPNL sont devenus des acteurs avec qui l'ensemble des acteurs associatifs du champ environnemental régional dont les ambitions dépassent l'échelle très locale doivent compter, que ce soit en recherchant leur appui ou en s'en distanciant. La montée en importance régionale de la SPNL résulte de la capacité de Sarhal à puiser dans plusieurs registres discursifs et à combiner plusieurs répertoires d'action adaptés à diverses échelles. Surtout, il a fait circuler les paradigmes entre échelles et en a proposé des traductions (la gestion participative devenant la hima) adaptées au cadre référentiel propre à chaque échelle. C'est ce travail d'adaptation et de traduction qui a permis l'hybridation de paradigmes globalisés avec des modèles locaux et qui a rendu possible leur adoption par de nouveaux acteurs du champ

$36 \quad$ KARAM K., 2004.

$37 \quad$ Entretiens avec A. Sarhal et des employés de la SPNL, 2010. 
environnemental arabe, les monarchies pétrolières du Golfe, qui cherchent à ancrer dans un référent islamique ce nouveau domaine de leur stratégie d'influence régionale.

\section{La Royal Society for the Conservation of Nature}

En Jordanie, la généalogie de la création et de la gestion des AP est inséparable du développement de la Royal Society for the Conservation of Nature (RSCN). La RSCN est précurseur régional (et parmi les précurseurs mondiaux) en matière de privatisation de la gestion des AP. En effet, l'idée, promue à partir des années 1980 par les agences internationales de développement, selon laquelle l'État n'est pas l'acteur idéal pour exécuter un certain nombre de tâches de service public a été anticipée dès la fin des années 1960 par le régime jordanien à qui manquait les moyens de ses politiques. Comme au Liban, mais avec une temporalité différente, ce n'est pas l'idée de décharge, impliquant une charge précédente, qui est pertinente ici, mais celle de délégation concomitante de la décision publique d'investir certains champs. En Jordanie, la gestion de la biodiversité a d'abord été conçue de manière restrictive autour de la régulation de la chasse, avec une expansion progressive de la sphère de délégation octroyée à la RSCN pour inclure la gestion des AP, puis le développement socioéconomique des populations vivant à proximité de ces zones après que la RSCN leur ait interdit l'accès aux ressources naturelles.

La RSCN a été créée en 1966 par Anis al-Mu'asher, un homme d'affaires jordanien qui occupera également plusieurs postes ministériels. Dans les années 1960, il est président du Royal Jordanian Hunting and Shooting Club et compagnon de chasse du roi Hussein. Ce sont les membres de cette élite jordanienne amatrice de chasse, inquiète de la raréfaction, voire de la disparition, du gros gibier, qui sont à l'origine de la création de la RSCN à laquelle le roi accorde son patronage ${ }^{38}$. La RSCN n'est donc pas à proprement parler une ONG royale comme il en existe plusieurs dans le pays, présidées (petit doute : vous faites référence à la RSCN ou les ONG ?) et non pas seulement patronnées, par un membre de la famille royale ${ }^{39}$. Elle a cependant, dès sa création, des canaux d'accès direct au Palais. En 1973, lui sont confiés deux mandats de service public, généralement prérogative des États : réguler la chasse en délivrant des permis de chasse et en créant les premières unités de gardes-chasses, mais aussi administrer les AP, conçues comme des réserves pour la protection ou la réintroduction 
d'espèces animales. La première réserve est ainsi créée à Shomari, près d'Azraq, en 1975 pour réintroduire l'oryx.

Dans les années 1990, alors que la gestion de la biodiversité émerge sur les agendas publics au Moyen Orient, la Jordanie a déjà une longueur d'avance tant en matière de planification que de législation. En 1980, elle a été l'un des tout premiers pays à s'engager à mettre en œuvre les recommandations de la Stratégie mondiale de la conservation préparée conjointement par l'UICN, le PNUE et le World Wildlife Fund (WWF) et qui a introduit pour la première fois le concept de développement durable. Un département jordanien de l'Environnement est créé en vue de proposer des projets de loi et d'élaborer et mettre en œuvre une Stratégie environnementale nationale (SEN). Déjà, la législation sur la protection de l'environnement prévoit le renforcement de la coordination et de la coopération avec les organisations de la société civile tant du secteur privé qu'associatif. La SEN, achevée en 1991, inclut une liste de douze zones prioritaires identifiées par un expert de l'UICN en vue de la création d'AP. La RSCN se voit confier ce mandat, appuyée par ce qu'Ingrid Schneider et G. Wesley Burnett, deux universitaires spécialistes de la gestion environnementale qui ont consacré un article à la gestion des AP en Jordanie, qualifient de «ministères et départements auxiliaires $»^{40}$. Le rôle du département de l'Environnement est en effet essentiellement de s'assurer que les lois sont appliquées et de coordonner et superviser les actions des partenaires associatifs et du secteur privé. L'État voit la délégation comme nécessaire, voire indispensable, à défaut d'avoir les moyens financiers de ses politiques ${ }^{41}$. Avec une assiette fiscale réduite, très dépendants de l'aide internationale qui commence à flécher des aides directes aux organisations de la société civile, les décideurs politiques jordaniens préfèrent ne pas dégager du budget public les sommes nécessaires à l'investissement dans les programmes de protection environnementale que le pays s'est engagé à mener en signant plusieurs conventions internationales.

Le champ public de l'environnement étant déjà largement structuré dès les années 1980, la ratification, en 1993, de la CBD par la Jordanie n'a pas été, comme au Liban, un catalyseur qui a donné une nouvelle impulsion à l'action publique dans ce domaine. Depuis les années 1990, sept nouvelles AP ont été créées, couvrant une superficie de $1200 \mathrm{~km}^{2}$. Fin 2010, neuf autres sont en cours de création ou en projet sur $2884 \mathrm{~km}^{2}$. La RSCN gère l'ensemble de ces réserves et a développé un très haut niveau de professionnalisation qui la rend attrayante pour les bailleurs, lesquels ont été très généreux en subventions à son égard. 
Cependant, le début des années 2000 marque un tournant dans l'attitude de l'État jordanien qui, comme d'autres gouvernements arabes, cherche à réduire l'accès d'organisations de la société civile revendicatrices à des sources de financement extérieures. Une série de mesures législatives sont adoptées qui rendent tout d'abord obligatoires l'approbation de ces financements par le Conseil des ministres, avant de culminer dans une décision obligeant les donateurs à faire transiter leurs subventions par le ministère du Plan et de la Coopération internationale qui en ponctionne une partie avant de les redistribuer. Ces mesures affaiblissent les ONG dont les activités sont le plus visées par le régime jordanien. Cependant elles touchent peu les ONG royales ou bien patronnées qui se partagent l'essentiel des champs de l'action sociale et environnementale. Ces ONG, sans doute également bien conseillées, ont en effet anticipé sur ces mesures et réformé leurs cadres financiers. C'est le cas de la RSCN qui, dès 2002, a créé un fonds de placement financé grâce à une subvention d'un montant de huit millions de dollars US octroyée par l'agence de développement des États-Unis (USAID). Les dividendes de ce fonds autorisent la RSCN à ne plus fonctionner uniquement sur projets mais à couvrir ses frais de fonctionnement à plus long terme, et donc à recruter du personnel et à développer ses activités et ses infrastructures. Par ailleurs, la RSCN continue d'être financée par des agences internationales sur projets, les fonds étant canalisés par le ministère du Plan.

Le recours à l'aide internationale et la recherche de l'autonomie financière par les placements financiers ne forment qu'un volet du développement institutionnel de la RSCN. Un autre volet est la marchandisation des AP par l'écotourisme et la production-vente de produits liés au thème de la nature. Depuis 1994 tout programme de protection environnementale est couplé avec des projets de développement socio-économique. Le projet phare en la matière est la réserve de $\operatorname{Dana}^{42}$ qui a bénéficié de financements de la GEF et de la Banque mondiale et de l'appui technique du PNUD. Le tournant que constitue Dana par rapport au modèle conservationniste précédemment utilisé dans les AP jordaniennes est justifié dans les documents de projet en référence à la gestion participative et au développement durable dans une région où il s'agit aussi bien de protéger la biodiversité que d'assurer des revenus à des populations pauvres car très dépendantes de salaires du secteur public en diminution relative dans les années $1990^{43}$. On peut alors interpréter le nouveau modèle gestionnaire adopté à Dana comme une décharge partielle du rôle économique de

42

Cette réserve est située dans le Sud jordanien entre Tafilé et Pétra.

C'est l'époque où le gouvernement jordanien, assujetti à un plan d'ajustement structurel imposé par le FMI, bloque les salaires et les retraites de la fonction publique tout en levant les subventions publiques sur plusieurs produits de première nécessité. Or dans la région de Dana, comme dans les autres régions rurales jordaniennes, une large part des actifs est employée par le secteur public. 
l'État sur le marché ${ }^{44}$. Dans ce processus, la RSCN est chargée d'une mission d'intermédiation entre les populations locales, qui sont contraintes de s'organiser en coopératives pour devenir des interlocuteurs légitimes, et une clientèle internationale et jordanienne citadine aisée sensible à l'environnement. Cependant, le rôle de la RSCN ne limite pas à la mise en relation d'opérateurs touristiques locaux avec une clientèle ni au marketing des AP. La RSCN se transforme en entreprise commerciale qui crée et gère, à Dana et dans d'autres AP, des unités de production artisanale ainsi que des services et des infrastructures touristiques (réception, accompagnement et transport des visiteurs, hôtellerie, campings, etc.). Par ce biais, la RSCN garde le contrôle des activités liées à l'écotourisme dans les AP où elle est en situation de quasi-monopole. Elle génère des revenus qui couvrent en grande partie les salaires des employés des unités de production et de ceux chargés des infrastructures et services touristiques. Ces revenus couvrent également les frais de gestion administrative des $\mathrm{AP}^{45}$.

On peut considérer que le processus de décharge du coût de la protection de la biodiversité dans les AP jordaniennes a connu deux étapes. Dans un premier temps, l'État a délégué ses fonctions de gestion à une association en faisant peser l'intégralité des coûts sur l'aide internationale. Dans un second temps, cette association s'est déchargée d'une partie des coûts de gestion sur le marché réel en transformant les populations locales en producteurs de biens et services. Cette seconde étape va par ailleurs dans le sens des politiques de réforme économique prônées par le FMI et la Banque mondiale, visant à réduire dans le marché de l'emploi la part du secteur public au bénéfice des entreprises privées ${ }^{46}$. Pour autant, s'il y a bien eu décharge des coûts de gestion, celle-ci n'a pas été accompagnée de la mise en place d'une cogestion entre la RSCN et les populations locales, ni d'une quelconque délégation d'autorité. Le modèle de Dana peut-être qualifié de participatif/consultatif, cependant les modalités d'administration et de prise de décision aussi bien à Dana que dans les autres AP où les communautés locales, sous la forme de coopératives, sont nominalement parties prenantes, sont en fait verrouillées, assurant à la RSCN une hégémonie totale sur les orientations, les décisions et la gestion des revenus financiers. En outre, l'association projette une image de puissance à travers des signes extérieurs ostentatoires: véhicules tous terrains et parc informatique dernier cri, bâtiments administratifs et d'accueil des visiteurs conçus et meublés par un architecte jordanien de calibre international, etc. Elle semble posséder une capacité

\footnotetext{
$44 \quad$ Anne Marie Baylouny (2010) arrive aux mêmes types de conclusion à partir d'une réflexion sur le désengagement de l'État jordanien des services sociaux.

$45 \quad$ Voir IRANI K. et JOHNSON C., 1998 et « The Nature Business », Jordan Business, 7 juillet 2008.

46 Schneider et Burnett soulignent l'une des dérives potentielles du modèle : le risque que les objectifs de rentabilité prennent le pas sur la protection environnementale (SCHNEIDER, I. et BURNETT G. W., 2000, p. 242).
} 
financière sans limites et le pouvoir de faire appuyer ses programmes et décisions par les autorités publiques, y compris lorsqu'il s'agit de réprimer les violations des règlements des réserves (braconnage, pâturage et coupe de bois illégaux, etc.). Il n'est pas étonnant, dans ce contexte, que la vision la plus répandue parmi les populations qui habitent à proximité des réserves soit que les RSCN sont une entité gouvernementale ${ }^{47}$.

Penchons-nous à présent sur les individus qui ont œuvré à la création du modèle Dana et à la transformation institutionnelle de la RSCN. L'acteur principal est un environnementaliste britannique, Chris Johnson, qui débute sa carrière à la RSCN en 1993 en tant qu'administrateur du projet de la GEF ayant pour objectif le renforcement des capacités institutionnelles de l'association. C'est Johnson qui introduit auprès des jeunes cadres jordaniens de formation scientifique les nouvelles approches et mots d'ordre internationaux de la gestion environnementale. Il parraine particulièrement un jeune diplômé de la faculté d'Agriculture, Khaled Irani, qui bénéficie d'une série de formations en gestion, dont certaines dans le domaine des AP. Nommé responsable du Département des réserves à la RSCN, Irani supervise la création de la réserve de Dana avant d'accéder au poste de Directeur général de la RSCN en 1996. Même dans cette fonction, Irani, qui n'est pas encore à l'aise pour s'exprimer en anglais, laisse à Johnson le rôle d'interface avec les interlocuteurs non arabes.

Johnson est le stratège du développement financier et institutionnel de l'association. Il assure dans un premier temps à la RSCN un accès continu à l'aide bilatérale et multilatérale en reconceptualisant les objectifs de l'association et en se chargeant de préparer les dossiers de demande de subventions. Nommé Directeur de la conservation au sein de la RSCN, Johnson recherche des partenariats avec des ONGI : la RSCN devient ainsi le premier partenaire de BirdLife au Moyen Orient puis accède au statut de membre de l'UICN en 2000. Enfin, Johnson prépare la transition de l'association vers les activités du secteur privé. Il est l'architecte des programmes socioéconomiques mis en place à Dana, et crée une unité écotourisme au sein de la RSCN. Quelques années plus tard, il crée et dirige Wild Jordan, le département commercial de la RSCN, dont l'objectif est de vendre les produits des AP et qui absorbe l'unité écotourisme. Une partie des bénéfices de Wild Jordan contribue à la gestion des AP et aux programmes de conservation. L'autre partie permet la croissance de Wild Jordan par le recrutement de personnel et la diversification des activités génératrices de revenus dans les AP. Le tout est inscrit dans une stratégie de financement qui inclut les dividendes de placements financiers et la recherche de mécénats d'entreprise.

$47 \quad$ Ces informations et celles qui suivent sont issues de plusieurs entretiens menés entre 1996 et 2010 avec Chris Johnson, d'autres membres du personnel de la RSCN, et des personnes vivant près des réserves. 
Johnson est un conservationniste radical qui voit les populations locales avant tout comme prédatrices des ressources naturelles ${ }^{48}$. Il ne croit pas dans un modèle d'AP autogérées par les populations locales mais dans une gestion techniciste par une autorité centrale dont il pense qu'elle est seule à même de dépasser les intérêts particularistes locaux et de faire imposer normes et réglementations. Il est peu sensible à un discours d'ancrage des modèles de gestion des AP dans des référents locaux, peut-être car il ne possède pas le capital linguistique et culturel qui lui permettrait de comprendre et traduire les concepts : il ne parle pas l'arabe et ses interactions avec les acteurs au siège de la RSCN comme dans les AP se limitent de ce fait à ceux qui ont absorbé le discours en anglais sur la biodiversité et le développement socioéconomique que leur a inculqué la RSCN. Il est donc difficile, au sein de la RSCN, de sortir du discours autoréférentiel, une tendance qui n'évolue qu'à partir du milieu des années 2000, on le verra plus loin.

À l'échelle nationale, d'autres dynamiques, dans lesquelles le rôle de Johnson n'est pas central, ont contribué à l'institutionnalisation de l'association. La situation de monopole dans laquelle se trouve la RSCN pour la gestion des AP tient historiquement de son patronage royal. Ce monopole n'est pas menacé après le décès du roi Hussein en 1999. En effet, le conseil d'administration de la RSCN se choisit un autre patron proche du régime en élisant à sa tête la sénatrice Laila Sharaf, ancienne ministre de l'information et épouse du Sharif Abdel Hamid Sharaf, un cousin du roi Hussein ayant occupé plusieurs postes dans la diplomatie et le gouvernement. Il ressort de nos entretiens avec d'autres ONG environnementales jordaniennes que celles-ci manquent de légitimité aux yeux des institutions publiques jordaniennes sur la question de la biodiversité. Elles n'arrivent pas non plus à créer un espace public de débat sur la gestion des AP qui pourrait faire émerger des modèles alternatifs. Pourtant une dizaine de ces associations sont dirigées par des individus possédant un capital éducatif de haut niveau, souvent acquis dans des universités anglo-saxonnes, et plusieurs entretiennent des connexions internationales en étant membres d'ONGI.

L'hégémonie de la RSCN dans le champ discursif national est rendue possible grâce aux liens étroits que l'association entretient avec les institutions publiques qu'elle a pénétrées de sa vision et son langage, et auxquelles elle a inspiré des modes organisationnels. Elle a participé à toutes les grandes étapes de la constitution d'un champ public de la protection environnementale, y compris l'élaboration d'une stratégie nationale et d'une législation et ce, au détriment du rôle joué par le département de l'Environnement. Lorsqu'est créé, en 2003, un

$48 \quad$ C'est ce qui ressort de plusieurs entretiens menés avec lui depuis 2001, sans que ses positions aient notablement évolué. 
ministère de l'Environnement (MdE), c'est Khaled Irani qui est chargé du portefeuille qu'il conserve jusqu'à 2009, une longévité remarquable dans le contexte jordanien où les remaniements ministériels sont parmi les plus fréquents du monde. En 2004, la délégation de gestion des AP octroyée à la RSCN depuis 1973 est formalisée par la signature d'une convention avec le $\mathrm{MdE}^{49}$. Aujourd'hui, les partenaires institutionnels publics de la RSCN comptent les ministères du Plan, du Tourisme, de l'Environnement, de l'Agriculture, de l'Éducation pour la création de programmes environnementaux dans le cursus scolaire, mais aussi la Police nationale pour l'application des lois sur la chasse et la protection de l'environnement ${ }^{50}$.

La RSCN, tout comme les ONG royales, participe du fonctionnement semi-rentier de l'État, dont l'une des sources de rente est l'aide internationale et, de plus en plus, le recours aux subventions du secteur privé et aux placements financiers. Elles constituent des entités qui, parce qu'elles ne représentent pas un potentiel contestataire et déstabilisateur pour le régime, sont autorisées à capter cette partie de l'aide internationale qui est fléchée pour les organisations de la société civile, tout en contribuant aux politiques publiques et en limitant les capacités d'autonomie financière et d'influence des autres ONG. Quintan Wiktorowicz avance que les ONG royales jordaniennes deviennent hégémoniques dans leurs domaines d'activités ${ }^{51}$, argument confirmé par le cas de la RSCN. Ces ONG sont également des matrices où se forme l'expertise en vue de positions publiques. La circulation des personnes entre cette branche du secteur associatif et l'administration maintient une homogénéité des référents, des modèles, et des méthodes dans les champs du développement et de l'environnement.

Les ONG royales peuvent être considérées comme des extensions de l'État. Cependant leurs activités sont rarement rentables ce qui n'est pas le cas de la RSCN. Celle-ci a su obtenir son autonomie financière en gagnant suffisamment de crédibilité auprès des bailleurs et en faisant payer le prix de la conservation aux marchés réel et financier, au point que sa relation à l'État et à l'action publique est beaucoup plus subtile. Elle a la capacité, qu'elle manie cependant avec prudence, de faire mettre sur agenda des questions relevant de son mandat sur les AP et a obligé le gouvernement à plusieurs reprises à revenir sur des décisions telles l'exploitation minière ou le développement hôtelier dans ces zones. Enfin notons que, forte de ses succès, la RSCN développe dès 1999 son expertise à l'échelle régionale, formant aussi

\footnotetext{
49 Le successeur d'Irani en tant que Directeur général de la RSCN, Yahyia Khaled, a également été formé et parrainé par Chris Johnson depuis le début des années 1990.

${ }_{50}$ Les deux premiers successeurs d'Irani à la tête du ministère de l'Environnement, qui ne sont pas des produits de la RSCN, ont tenté de limiter le champ d'action de l'ONG, en particulier en s'opposant à l'extension du territoire sous son contrôle par la création de nouvelles AP. 51

WIKTOROWICZ Q., 2002, p. 85.
} 
bien au Liban qu'en Syrie une partie de la nouvelle génération de professionnels de la protection environnementale, et agissant en tant que consultant pour la mise en place de nouvelles AP de la génération participative/consultative et ouvertes au marché sur le modèle de Dana.

Si la RSCN a anticipé sur le modèle des années 1980 de privation de la gestion des ressources naturelles et a été précurseur dans l'introduction d'un volet socio-économique à Dana, elle a, dans les années 2000, du mal à négocier le tournant de la gestion directe par les communautés locales. Ce volet nécessiterait non seulement des réformes en profondeur de son organisation interne mais également un retour sur le paradigme conceptuel qui a assuré son hégémonie dans le cadre national. Pourtant, la RSCN envisage d'introduire un modèle proche de celui de la hima dans certaines des nouvelles AP qui sont en cours de création dans la vallée du Jourdain, en tentant toutefois de conserver un rôle décisionnaire. Notre hypothèse est qu'elle voit ce modèle de gestion comme une décharge supplémentaire sur la communauté locale. Il va s'agir, en chargeant de la gestion administrative les membres des coopératives locales qui ne seront pas salariés par la RSCN, de réduire les coûts de personnel de l'association qui sont devenus trop lourds. Début 2011, la RSCN salarie en effet plus de 320 personnes dans ses deux centres à Amman et dans les AP. Non sans ironie, le développement institutionnel de la RSCN l'a conduit à une situation similaire à celle des institutions publiques jordaniennes qui, face à un nombre de fonctionnaires dont elles ne peuvent plus supporter les charges salariales, privatisent des pans entiers du secteur public et font appel à la soustraitance.

Il n'est pas sûr, cependant, que la RSCN décide d'adopter l'appellation de hima dans les nouvelles AP. En effet, la RSCN s'est vue concurrencée et en partie distanciée par la SPNL dans son ambition de créer un modèle régional. Aussi, la direction de la RSCN hésite à adopter un concept dont elle ne peut revendiquer la paternité. D'un autre côté, il faudra voir si l'association résiste à l'attrait des financements offerts par les fonds hima-s régionaux qui promettent d'être bien dotés. Enfin, une nouvelle génération d'environnementalistes jordaniens a accédé à des postes de responsabilité au sein de l'association au cours des années 2000 : ces trentenaires, dont plusieurs ont été formés dans les universités de pays occidentaux, contestent la vision conservationniste qui a prévalu jusqu'ici et sont également sensibles aux nouveaux branchements entre éthique islamique et protection environnementale. 


\section{La Syrian Society for the Conservation of Wildlife}

En Syrie, les dynamiques politiques et bureaucratiques nationales ont pesé plus lourd que l'agenda international dans le calendrier et la nature de la structuration du secteur public de l'environnement. On peut faire remonter le début de ce processus aux années 1990 lorsque les autorités syriennes, dans le cadre de la nouvelle infitah (ouverture) pensée en réponse à la crise de l'économique étatisée ${ }^{52}$, sollicitent la Banque mondiale pour un soutien technique à la formulation d'un Plan d'action environnementale (le Programme 21 Syrie). En 1991, est nommé pour la première fois un ministre d'État pour les Affaires environnementales. Dans les années qui suivent, la responsabilité de l'environnement est transférée au ministère de l'Administration locale qui devient par la suite ministère de l'Administration locale et de l'Environnement (MALE).

La situation en matière d'autorité sur les AP syriennes ainsi que le statut des ces zones sont complexes. L'appellation mahmiya (réserve) recouvre différents types d'AP qui relèvent de deux ministères - le ministère de l'Agriculture et de la Réforme agraire (MARA) et le MALE - dont les responsabilités se chevauchent sur les mêmes territoires. Plusieurs types d'AP existent : vingt-six réserves naturelles, seize forêts domaniales, des réserves de chasse, des zones de reproduction du gibier, et soixante zones de protection des pâturages. Ces réserves ne sont pas alignées sur les standards de l'UICN qui sont indispensables pour recevoir des financements des organisations internationales spécialisées (l'aide bilatérale est plus souple dans ce domaine). De plus, la plupart des zones classées mahmiya au Journal officiel n'ont aucune structure de gestion : au début des années 2000, sur les vingt-six réserves naturelles classées, seules deux étaient dotées d'une structure administrative ${ }^{53}$. Comme dans une majorité de pays du Sud, la philosophie qui a guidé l'établissement et l'administration de ces réserves est celle de l'interventionnisme étatique. Ce modèle est techniciste, centralisateur et vise à protéger les ressources pour en assurer une meilleure exploitation. Le classement en AP se fait par décision ministérielle sans aucune consultation locale. Les activités humaines sont alors en théorie interdites. Mais malgré l'inflation réglementaire, les pouvoirs publics sont incapables de faire appliquer leurs décisions. En outre, les arrangements institutionnels verrouillent la souveraineté de l'État sur ces territoires et rendent difficile l'implication 
d'acteurs non gouvernementaux qui pourraient bénéficier de délégations ${ }^{54}$.

La Syrie ratifie la CBD en 1996. La même année, le gouvernement crée une Unité nationale pour la biodiversité (UNB) au sein du MALE. L'UNB est responsable des AP et se déploie sur le territoire syrien à travers quatorze Directorats de l'environnement au sein de l'administration locale des gouvernorats. Cependant, les prérogatives de l'UNB empiètent sur celles du MARA et conflits d'autorité et procédures bureaucratiques complexes (nationales et au niveau des gouvernorats) obèrent la plupart des tentatives d'améliorer la gestion des AP et de faire appliquer la loi ${ }^{55}$. En outre, il n'y pas, dans les administrations syriennes, de personnel formé aux approches et méthodes de la gestion environnementale promues par les institutions internationales $^{56}$. Enfin, en 1996, la Banque mondiale, principal partenaire de la GEF, n'a pas de programme de prêt destiné à la Syrie et le pays n'est membre d'aucune des grandes banques régionales de développement autorisées à gérer les prêts octroyés par la $\mathrm{GEF}^{57}$.

En 1998, alors que la Syrie recommence à faire appel à la Banque mondiale après un hiatus de treize ans, la GEF octroie une première subvention de 750000 dollars US, couvrant la période 1999 à 2004, à un projet de protection de la biodiversité et de gestion des AP. Il s'agit de renforcer les capacités institutionnelles du MALE et du MARA et de développer un projet pilote de gestion des AP dans la réserve de Slounfé ${ }^{58}$ qui puisse servir de modèle à reproduire dans d'autres $\mathrm{AP}^{59}$. Selon la Banque mondiale, le projet est un échec dû à des «blocages institutionnels » et à la lenteur de la procédure de financement due au manque de familiarité du pays avec les procédures de la Banque ${ }^{60}$. Sur le terrain, les personnes impliquées parlent autant de détournements de fonds et de corruption que de conflits d'autorité entre le MALE et MARA ${ }^{61}$. Sans doute pour toutes ces raisons, le PNUD et l'Organisation des Nations Unies pour l'alimentation et l'agriculture (FAO) ont retiré leur offre initiale de cofinancement qui devait, entre autres, permettre la mise en place d'activités génératrices de revenus pour les populations locales sur la base de microcrédits.

Au-delà des « blocages » administratifs, on peut aussi avancer qu'il n'y a guère, à la fin des années 1990, de volonté politique pour pousser à des réformes législatives ou régulatrices dans le domaine environnemental. Comme dans d'autres secteurs du développement en

\footnotetext{
54

54 Observations de terrain et entretiens dans plusieurs AP, 2007.

55 Entretiens à l'UNB et avec trois responsables d'AP, 2007.

56 PNUD, s. d. et DECS, 2009.

$57 \quad$ GEF, 2008 et DECS, 2009.

58 Cette réserve est située dans le Jebel Ansariye.

59 BANQUE MONDIALE, 2003b, p. 591.

60 BANQUE MONDIALE, 2003b, p. 591.

61 Entretiens avec d'anciens consultants, l'ancien responsable de la réserve de Slounfé et des habitants de la région, 2006 et 2007.
} 
Syrie $^{62}$, c'est l'arrivée au pouvoir de Bachar Al-Assad qui marque des changements d'orientation impulsés par le régime ${ }^{63}$ : une loi de protection environnementale, adoptée en 2002, prévoit la création d'un fonds pour soutenir les politiques publiques dans ce domaine ; également, une stratégie et un plan d'action nationaux pour l'environnement, insistant sur le développement durable, sont lancés en 2003 pour couvrir la période allant jusqu'en 2010. Ce programme sectoriel est aligné sur les objectifs du $10^{\mathrm{e}}$ plan quinquennal (2006-2010) qui introduit le concept d'économie sociale de marché et ouvre la porte à l'implication de la société civile (ONG et secteur privé) dans les mécanismes de décision et d'application des politiques publiques.

Pour dépasser les blocages entre le MALE et le MARA en utilisant le nouveau cadre programmatique et législatif qui est en train de se mettre en place, l'UNB, principal architecte de la stratégie et du plan d'action nationaux pour l'environnement de 2003, propose comme solution d'associer les « communautés locales » à l'administration des AP tout en prenant en compte les besoins économiques des populations. C'est pour l'aider à faire appliquer ce nouveau programme que la Syrie fait à nouveau appel aux bailleurs internationaux. En 2005, un nouveau projet de plus de 4 millions de dollars US prévu pour sept ans -- financé par la GEF et le PNUD avec participation syrienne-- relance la conservation de la biodiversité et la gestion des $\mathrm{AP}^{64}$. Cette fois-ci, une unité de gestion de projet indépendante du MALE et du MARA est mise en place, officiellement pour faciliter la coordination entre les deux ministères mais également, officieusement, pour limiter les détournements de fonds ${ }^{65}$. Entre 2005 et 2010, des subventions plus modestes sont octroyées par l'aide bilatérale (dont l'agence suisse de coopération), ou multilatérale (Commission européenne, FAO, etc.) pour la transformation des modes de gestion d'AP existantes. Tous ces projets adoptent le modèle participatif et gestionnaire ainsi que le discours du développement durable qui implique la création d'activités économiques de substitution pour les populations locales.

C'est sur le terrain de ces activités économiques, tout particulièrement l'écotourisme, que les ONG peuvent espérer obtenir des délégations de l'État. Les années 2000 représentent en effet un tournant pour le secteur des ONG en Syrie ${ }^{66}$. De nouveaux types d'associations,

\footnotetext{
62 Voir LE SAUX M., 2006 et RUIZ DE ELVIRA L., 2010.

63 Sur l'économie politique du développement en Syrie sous Hafez puis Bachar Al-Assad, voir PERTHES V., 2007 ainsi que HINNEBUSCH R. et SCHMIDT S., 2008.

64 PNUD, s. d.

65 Le PNUD et la GEF imposent des procédures comptables strictes dont l'accord du responsable au projet au sein du PNUD pour toute dépense dépassant un montant plafonné. Entretiens avec des responsables du projet au PNUD et à l'unité de gestion du projet, 2007.

Selon Le SAUX M., 2006 et RuIZ DE Eliva L., 2010.
} 
indépendantes des structures du parti Ba'ath, sont autorisées à s'enregistrer auprès du ministère du Travail et des Affaires sociales dans des secteurs que le pouvoir considère comme non politiques, ce qui est le cas de l'environnement. En 2009, il existe une quarantaine d'ONG enregistrées mentionnant l'environnement dans leurs statuts. Plusieurs ont un champ d'action local et multisectoriel. La plupart a un nombre de membres restreint et n'a pas la capacité de mettre en œuvre des projets ou de lancer des campagnes d'envergure ${ }^{67}$.

La Syrian Society for the Conservation of Wildlife (SSCW) est l'une des toutes premières ONG à vocation nationale et strictement environnementale créées en Syrie. L'initiative voit le jour sous l'impulsion du PNUD qui, au vu de l'échec de l'expérience de la réserve de Slounfé, rassemble une équipe d'experts syriens pour préparer le nouveau projet de protection de la biodiversité et de gestion des AP qui sera lancé en 2005. Au cours de cet exercice, qui familiarise les participants syriens avec les nouveaux modèles de délégation de la gestion environnementale à la société civile, un petit groupe de personnes décide de créer la SSWC. Parmi les fondateurs on trouve le Dr Akram Darwish, directeur de l'UNB, et Osama al-Nouri, propriétaire d'une importante agence de voyages qui a participé à l'étude du PNUD en tant que consultant sur la question de l'écotourisme ${ }^{68}$. Se saisissant du vocabulaire du développement durable, la SSWC se donne pour mission : «To protect the wildlife, its natural ecosystems, habitats and environment in a balanced and sustainable manner through gaining internal, regional and international support to respond to the needs of all humanity and to link that to local development programs within a constructive and participatory approach ${ }^{69}$.

Darwish assume la présidence de l'association et Nouri son secrétariat général. Ils allient leurs compétences et leurs réseaux : Darwish est un scientifique, Nouri est titulaire d'un MBA obtenu dans une université américaine ; Darwish, par ses fonctions professionnelles, est situé au cœur de l'administration centrale et du secteur public de l'environnement, tandis que Nouri est un proche du président Bachar Al-Assad, auprès de qui il assure les fonctions d'interprète lors de visites officielles; il est par ailleurs connecté au secteur national et international du tourisme. Les deux hommes se partagent stratégiquement les actions en vue d'institutionnaliser l'association au niveau national et international. Darwish a étudié en Syrie et ne parle pas anglais, tandis que Nouri possède les codes sociaux, les compétences linguistiques et les capacités de mobilité internationale qui lui permettent d'évoluer au sein

\footnotetext{
$67 \quad$ DECS, 2009.

68 Entretiens avec A. Darwish et O. Nouri, 2007 et 2008.

69 Selon son embryon de site internet en anglais consulté en décembre 2010 : http://wacsi.unm.edu/affiliates/sscw-syrian-society-for-the-conservation-of-wildlife. Le site arabe est plus développé : http://www.sscw-syria.org/ar/ mais formule la mission de l'association en des termes semblables.
} 
des ONGI et de leurs forums.

Alors que, selon Mathieu Le Saux, les ONG créées par Mme Asma Al-Assad, épouse du président syrien, utilisent leur proximité avec le pouvoir comme source de crédibilité auprès de bailleurs de fonds, dans le cas de la SSWC, la proximité du pouvoir ne semble pas suffire ou ne pas être assez étroite. Il est certain, que, comme l'écrit Le Saux «Face à [un] agenda initié "par le haut", la création et l'officialisation d'associations ne pouvant se prévaloir d'un rapport aussi direct avec les membres des cercles au pouvoir demeure une chose difficile en Syrie, tant le cadre autoritaire marque sa présence dans un champ qu'il n'entend pas laisser à lui-même ${ }^{70}$. Cependant, créer une association n'est pas l'institutionnaliser en développant son champ d'action. Pour passer à cette étape, les relations personnelles de Nouri avec la famille au pouvoir ne suffisent pas car il faut convaincre des acteurs institutionnels qui sont situés hors de la sphère de pouvoir syrienne et auprès desquels il peut être contre-productif de se prévaloir de relations personnelles avec le Président. Il faut donc aussi faire montre de compétences techniques, de capacité à manier des langages et des concepts, et s'assurer une visibilité dans le champ international pour gagner la crédibilité nécessaire à l'octroi de responsabilité de programme et financement de la part aussi bien des pouvoirs publics syriens que des bailleurs internationaux 71.

La SSWC développe sa légitimité autour de deux thématiques: la protection des oiseaux et l'écotourisme. Sous la direction de Darwish, l'association publie le premier guide des oiseaux de Syrie en arabe, un ouvrage coproduit par BirdLife et cofinancé par la Banque mondiale. De manière assez attendue, la SSWC devient le partenaire syrien de BirdLife en 2010. De son côté, Nouri se place dans les ONGI et les institutions internationales de financement en jouant de ses deux compétences. En tant que propriétaire d'une agence de voyages, il est nommé unique représentant du secteur privé au comité de suivi syrien des «GEF small grants projects» qui alloue des subventions aux ONG à hauteur de 50000 dollars US. En tant que secrétaire général de la SSWC, qui est devenue le premier (et, début 2011, le seul) membre syrien de $1^{1} \mathrm{UICN}^{72}$, il est nommé trésorier de sa Commission régionale pour l'Asie occidentale.

\footnotetext{
$70 \quad$ LESAUX M., 2006, p. 200.

71 Entretien avec O. Nouri, 2008. Ces remarques sont tout autant valables pour les ONG sous patronage de la Première dame syrienne, dont les cadres font partie de l'élite globalisée arabe (ils ne sont pas tous syriens), ont étudié dans les pays occidentaux, parlent parfaitement l'anglais et se tiennent au courant des débats dans la sphère internationale du développement. Entretiens et observations, Syria Trust for Development, 2007-2010. Voir également la contribution de Claudie Fioroni dans ce volume.

72 Contre sept ONG libanaises et onze ONG jordaniennes en plus de l'Université de Jordanie et du MdE jordanien.
} 
Nouri et Darwish concentrent leurs efforts sur deux AP qui ne sont pas concernées par le programme du PNUD en cours. Ces AP bénéficient du classement par BirdLife en tant que zones importantes pour la conservation des oiseaux. L'une est située à Jabboul, dans la région d'Alep, l'autre est la réserve de Talila, à l'est de Palmyre. Dans les zones rurales où sont situées les AP, des coopératives locales ont été créées à partir des années 1960 par l'État ba'athiste socialiste dans le cadre de la réforme agraire ou du développement de l'agropastoralisme dans les steppes. L'État cherche une nouvelle fonction pour ces coopératives dans un contexte de crise économique et de réforme de l'économie étatique. Dans les deux AP, la SSWC a d'abord collaboré à des études de faisabilité sur l'écotourisme financées par BirdLife ou l'UICN. Dans un second temps, la SSWC recherche des financements pour lancer des projets portés par l'association avec un volet participatif, les coopératives devant être les partenaires locaux du projet. En moins de trois ans, la SSWC obtient une subvention de la GEF pour un projet de protection des espèces aviaires menacées à Jabboul, puis un autre financement octroyé par l'agence de coopération suisse pour le développement de l'écotourisme en collaboration avec la coopérative locale ${ }^{73}$. Ceci fait de la SSWC l'une des deux seules ONG environnementales syriennes bénéficiant de subventions directes de la part d'un bailleur bilatéral européen ${ }^{74}$. Comme il est indispensable pour une association syrienne d'obtenir l'aval du ministère du Travail et des Affaires sociales afin de recevoir des fonds étrangers, on peut avancer sans grand risque que ce privilège signale la proximité du secrétaire général de la SSWC avec le régime.

Les relations de Nouri avec la famille présidentielle jouent un rôle certain dans le cas de la réserve de Talila qui fait l'objet de l'attention particulière de Mme Al-Assad : y survit une petite colonie d'ibis chauves du désert, une espèce qu'on croyait éteinte en Syrie, et que la Première dame syrienne a décidé de sauver en patronnant la réserve créée en 2004 pour favoriser leur reproduction ${ }^{75}$. Ce sont avant tout ces relations qui ont permis à la SSWC d'apparaître, dans l'étude sur l'écotourisme financée par BirdLife (Serra 2007), comme le partenaire naturel et unique du secteur associatif national. Début 2011, la SSWC était en discussion avec le MALE pour obtenir l'exclusivité du développement de l'écotourisme à Talila. Il s'agirait pour la SSWC de jouer le rôle d'intermédiaire entre les différents acteurs (ministères, coopérative locale, secteur privé et BirdLife) impliqués dans le développement de

\footnotetext{
73 Ce projet est mené en collaboration avec la RSCN et vise à reproduire le modèle de la réserve jordanienne de Dana. 
l'écotourisme. Elle devrait également former les membres de la coopérative afin qu'ils acquièrent les compétences techniques nécessaires à une gestion locale de la réserve, de les aider à développer des produits touristiques et de leur permettre l'accès au marché du tourisme national et surtout international. Enfin, la SSWC aurait un rôle de conseil auprès du MALE en vue de développer un appareil de régulation et de certification de l'écotourisme à l'échelle nationale $^{76}$. Dans le cas où le MALE donnerait son aval au projet, l'UICN et BirdLife ont assuré la SSWC de leur appui technique et de leur aide à la recherche de financements internationaux ${ }^{77}$. Comme en Jordanie, des discussions sont en cours sur la pertinence d'adopter l'étiquette hima pour la réserve de Talila, qui permettrait d'accéder aux financements des fonds régionaux spécialisés mais qui a mauvaise presse dans les steppes et l'administration syriennes depuis l'échec des coopératives agropastorales organisées sur ce modèle $\mathrm{e}^{78}$.

Nous ne sommes pas ici dans le «contournement » évoqué par Le Saux pour « utiliser et jouer des nouveaux espaces d'autonomie constitués $»^{79}$ par l'ouverture de champ associatif syrien. Bien au contraire, la SSWC est au cœur et à l'avant-garde des logiques de réforme de l'action publique portée par le régime en vue d'enrôler les secteurs associatif et privé dans un partenariat avec l'État, tous ces acteurs étant conjointement engagés dans la mobilisation de soutiens internationaux. Pourtant, alors que la SSWC est devenue en quelques années le partenaire syrien privilégié des organisations internationales dans le champ de la protection de la biodiversité, début 2011 l'association n'a toujours aucun employé à plein temps, elle utilise les ressources humaines de l'agence de voyages de Nouri et de l'UNB et elle est domiciliée à la même adresse que ladite agence de voyages. La SSWC n'est-elle pas plutôt la façade associative d'une entreprise du secteur privé ? Ou bien sert-elle aussi les intérêts du MALE qui cherche, à travers l'UNB, à assurer sa suprématie sur le MARA en matière de gestion de la nouvelle génération d'AP qui sont en plein développement? En Syrie, la gestion des AP est un domaine de l'action publique en pleine mutation où la compétition pour les ressources est vive entre administrations publiques. L'ouverture de ces zones au marché, via l'écotourisme, en fait également un secteur qui intéresse plusieurs agences de voyages syriennes. Dans un tel donc un sujet sensible où le pouvoir impose ses règles. Là aussi, néanmoins, des pratiques de contournement existent : elles peuvent prendre la forme de coopération avec des ONG internationales (Movimondo, fondation Aga Khan...) ou des ambassades qui endossent la responsabilité officielle du projet, l'association se cantonnant au rôle de partenaire » (LE SAUX M., 2006, p. 205).
} 
contexte, la forme associative peut apparaître comme un outil privilégié pour répondre à l'offre de ressources et aux préoccupations des bailleurs internationaux et des ONGI qui veulent faire avancer l'agenda de la biodiversité dans le pays. Cette forme peut sembler extérieure tant à l'administration publique (dont l'image est celle d'une bureaucratie inefficace et corrompue), qu'au secteur privé (dont les intérêts particularistes peuvent apparaître comme contraires à la préservation des biens publics environnementaux).

Cependant, l'exemple de la SSCW pose la question de l'autonomie réelle du champ associatif syrien $^{80}$. En effet, la SSCW n'a aucune substance hors de sa relation avec le secteur public et le secteur privé. Ou plutôt, elle n'a pas de réalité hors des actions de ses deux principaux promoteurs qui l'utilisent, en mutualisant leurs ressources, comme vecteur de captation de capital social, financier et économique pour améliorer leurs positions respectives dans l'administration et sur le marché du tourisme. Dans le cas de la SSCW, les relations personnelles et d'avantages mutuels entre acteurs du secteur public et privé, doublées du patronage présidentiel, apparaissent comme les conditions nécessaires à la réalisation d'un début de décharge sur le secteur associatif de prérogatives de l'État syrien en matière de développement économique dans les zones rurales.

\section{Conclusion}

Face aux nouvelles injonctions internationales de développement durable et de protection de la biodiversité, qui pénètrent le champ environnemental arabe dans les années 1990, l'approche sociohistorique de trois ONG environnementales dans leurs temporalités et contextes propres permet de faire ressortir un certain nombre d'éléments relatifs aux configurations entre acteurs publics et associatifs et aux politiques d'échelles dans le champ de la protection de la biodiversité.

Au Liban, l'action publique en matière de gestion des AP s'est structurée dans les années 1990, soit celles de l'après-guerre civile, en plusieurs mouvements concomitants : la création d'institutions publiques régulatrices, la délégation de tâches de gestion aux acteurs municipaux, et la délégation de fonctions d'intermédiation entre municipalités, État, bailleurs et marché à des ONG nationales gestionnaires bénéficiant dès le départ d'une légitimité internationale. Enfin, le secteur des ONG environnementales était lui aussi en voie d'institutionnalisation lorsque l'action publique s'est intéressée à la gestion des AP. En outre, par d'autres types d'ONG de tentatives de contournement ou de préservation d'un espace associatif propre. 
l'administration publique libanaise, tout comme le secteur associatif, est investie par des logiques de patronages multiples. Aussi, l'État n'a pas donné le monopole de l'intermédiation à une seule ONG dans l'ensemble des AP. Dans ce contexte compétitif, la SPNL a dû déployer une stratégie à plusieurs échelles, manier plusieurs répertoires discursifs, faire preuve de sa légitimité gestionnaire, le tout en vue d'accumuler des ressources très diverses pour obtenir une part des mandats d'intermédiation.

En Jordanie, ce n'est pas tant l'action publique qui a présidé à la création des AP, que la volonté royale s'appuyant sur une ONG et ce, dès le début des années 1970. La délégation de responsabilité gestionnaire à la $\mathrm{RSCN}$ a précédé le développement d'institutions et de politiques environnementales publiques que l'association a fortement influencées. En situation de monopole gestionnaire et d'hégémonie discursive en Jordanie, la RSCN s'est construit une légitimité nationale qui lui a permis de se projeter à l'échelle supranationale en obtenant des financements et en devenant membre d'ONGI lorsque ces organisations internationales ont cherché à étendre leur influence dans la région. Dans les années 1990, du fait de son inscription dans ces réseaux internationaux, la RSCN a dû se faire l'écho du changement de paradigmes en matière de protection de la biodiversité et de gestion participative. Aussi, elle a introduit un nouveau modèle de gestion des AP qui l'a amené à effectuer une décharge de ses coûts de gestion sur le marché de l'écotourisme. En sortant de son rôle gestionnaire pour développer des activités entrepreneuriales, elle s'est trouvée investie par l'État d'un rôle de substitution: la création d'emplois et de richesses pour les populations rurales. La RSCN cherche à présent à se décharger à son tour de ce rôle sur des acteurs locaux au sein des AP.

En Syrie, l'environnement est un domaine que l'administration publique a investi de manière exclusive dès les années 1970. Les années 2000 marquent un début de rupture avec le modèle étatique. Les réformes impulsées par le régime ont plusieurs effets : elles touchent le secteur des ONG qui commence à se développer ; elles introduisent la gestion participative dans les AP via des associations locales et nationales ; enfin elles ouvrent davantage la porte aux bailleurs internationaux et aux nouveaux modèles que ces bailleurs promeuvent. Dans les AP, le régime syrien cherche en même temps à améliorer la gestion administrative que les institutions publiques n'ont jamais pu assurer, à décharger l'État du développement économique qu'il n'est plus en mesure d'assumer, mais également à continuer à maintenir un contrôle politique sur le territoire et les acteurs. La SSCW répond à toutes ces conditions : c'est un vecteur associatif pour des acteurs qui sont inscrits dans le secteur privé, mais également dans les réseaux de patronage présidentiel ; c'est également un outil qui devrait permettre de contourner les blocages issus de conflits entre administrations publiques en 
permettant au MALE d'assurer sa suprématie sur le MARA. C'est à partir de ressources puisées dans ces trois espaces relationnels (secteurs privé et public, et patronage présidentiel) que la SSCW a assuré son accès aux ONGI et aux financements internationaux. Ce début de décharge contrôlée de l'État sur le secteur associatif, et le rôle de catalyseur de la réforme de l'administration publique qu'il veut lui faire jouer, ne concernera sans doute qu'une poignée d'ONG ayant des liens forts avec le pouvoir et le secteur privé et qui ne seront pas en concurrence avec d'autres ONG dans leurs domaines d'activité.

Dans aucun des cas étudiés les acteurs associatifs ne se positionnent dans des logiques de concurrence par rapport à l'action publique. Plutôt ils jouent un rôle complémentaire de celui de l'État. Les ONG dont il a été question ici inscrivent leurs actions dans le paradigme néolibéral. Leurs actions sont certes contraintes par la demande publique, soit la recherche de ressources financières en dehors de l'espace national via les marchés globalisés (réel et/ou financier) et les bailleurs internationaux. Cependant, les acteurs principaux au sein de ces ONG adhèrent pleinement à une écologie de marché qu'ils ne perçoivent pas comme allant à l'encontre de leurs propres démarches, convictions et intérêts. Pour ces ONG gestionnaires, le recours au supranational n'est pas une manière de contourner l'État mais d'acquérir des ressources afin de se faire reconnaître comme partenaire légitime dans la formulation et/ou la mise en œuvre de politiques qui restent avant tout publiques.

À un autre niveau, la tension entre conditions posées par les États et attentes des partenaires ou bailleurs internationaux peut être un catalyseur permettant la construction d'une nouvelle échelle régionale où puiser ressources et légitimité. La SNPL et quelques autres acteurs dont on n'a pas pu traiter dans ce chapitre, en développant un discours et des référents adaptés à l'échelle régionale, ont créé un nouveau champ en voie d'institutionnalisation qui se structure autour d'ONG, de bailleurs, de programmes et de nouveaux paradigmes. Les acteurs de ce champ régional ambitionnent de peser sur les agendas nationaux ce qui amènera sans doute à de nouvelles configurations dans les relations entre ONG, États et secteur privé.

Pour finir, il nous semble que l'exemple de ces trois ONG invite à repenser la typologie classique utilisée pour rendre compte de la diversité des formes associatives au Moyen Orient (associations caritatives, de développement, de plaidoyer, ONG gouvernementales, ONG royales, etc.). Il faudrait sans doute y adjoindre ces ONG hybrides issues de l'expansion géographique et sectorielle du modèle néolibéral. Pour l'heure, il est néanmoins difficile de proposer une nouvelle typologie stable tant ces ONG sont plastiques et dynamiques. 


\section{Bibliographie}

ANHEIER Helmut, 2005, Nonprofit Organizations: Theory, Management, Policy, New York, Routledge.

ABU-SADA Caroline, 2007, ONG palestiniennes et construction étatique, Beyrouth, Institut français du Proche-Orient.

BANQUe MONDIALE, 2003a, Cornerstones for Conservation: World Bank Assistance for Protected Areas. Paper was prepared for the Fifth World Parks Congress in Durban, 2003, Washington, DC, Banque mondiale.

Banque mondiale, 2003b, Report on the Status of Projects in Execution, Operations Policy and Country Services, April 2003, Washington, DC, Banque mondiale.

Baylouny Anne Marie, 2010, Privatizing Welfare in the Middle East: Kin Mutual Aid Associations in Jordan and Lebanon, Bloomington, Ind., Indiana University Press.

Ben Nefissa Sarah, AbD AL-FatTah Nabil, Hanafi Sari et Milani Carlos (dir.), 2004, ONG et gouvernance dans le monde arabe, Paris, Karthala et Le Caire, CEDEJ.

Biggs S. et NEAmE A., 1995, « Negotiating Room for Manoeuvre: Reflections Concerning NGOs Autonomy and Accountability Within the New Policy Agenda », in EDWARDS M. et Hulme D. (dir.), Non-Governmental Organisations. Performance and Accountability. Beyond the Magic Bullet, Londres, Earthscan Publications, p. 31-40.

Bocco Riccardo, 1993, «De la sécurité politique à l'autonomie alimentaire dans les steppes : experts internationaux et programmes de développement (1950-1990) », in BoCCO Riccardo, JAUBERT Ronald et METRAL France (dir.), Steppes d'Arabie. États, pasteurs, agriculteurs et commerçants : le devenir des zones sèches, Paris, Puf et Genève, IUED, p. 326-357.

BouRdiEU Pierre, 1980, «Le capital social. Notes provisoires », Actes de la recherche en sciences sociales, 31 , p. 2-3.

BOURDIEU Pierre, 1994, Raisons pratiques. Sur la théorie de l'action, Paris, Éditions du Seuil.

BRAND Laurie, 2001, «Development in Wadi Rum? State Bureaucracy, External Funders, and Civil Society », International Journal of Middle East Studies, 33-4, p. 571-590.

CAMAU Michel, 2002, «Sociétés Civiles "Réelles" et Téléologies de la Démocratisation », Revue Internationale de Politique Comparée, 9-2, p. 213-232.

Challand Benoît, 2009, Palestinian Civil Society: Foreign Donors and the Power to Promote and Exclude, Londres, Routledge.

Chartier Denis et Ollitrault Sylvie, 2006, « ONG et développement durable : les liaisons dangereuses », in AUBERTIN Catherine et VIVIEN Franck-Dominique (dir.), Le 
développement durable, enjeux politiques, économiques et sociaux, Paris, La Documentation française, p. 93-114.

Chatelard Géraldine, 2008, Jebel Abdel Aziz Socioeconomic Assesment, Rapport non publié pour l'UNDP Syrie dans le cadre du projet "Biodiversity Conservation \& Protected Area Management".

Chatelard Géraldine, 2005, «Desert tourism as a substitute for pastoralism? Tuareg in Algeria and Beduin in Jordan », in CHATTY Dawn (dir.), Nomadic Societies in the Middle East and North Africa: Entering the $21^{\text {st }}$ Century, Leiden, Brill, p. 710-736.

Chatelard Géraldine, 2003, «Conflicts on interests over the Wadi Rum reserve: were they avoidable? A socio-political critique », Nomadic Peoples, 7-1, p. 138-158.

DECS (2009) Country Environmental Profile for the Syrian Arab Republic, Damas, Délégation de la Commission européenne en Syrie.

EIKENBERRY Angela et DrAPAl Kluver Jodie, 2004, «The Marketization of the Nonprofit Sector: Civil Sociely at Risk? », Public Administration Review, 64-2, p. 132-140.

FolTZ Richard, DENNY Frederick, et BAHARUdDin Azizan (dir.), 2003, Islam and Ecology: A Bestowed Trust, Cambridge, Mass, Harvard University Press.

FROGER Géraldine et ANDRIAMAHEFAZAFY Fano, 2003, «Les stratégies environnementales des organisations internationales dans les pays en développement: continuité ou ruptures ? », Mondes en Développement, 31-124, p. 49-76.

GARI Lutfallah, 2006, «A History of the Himā Conservation System », Environment and History, 12, p. 213-28.

GEF, 2009, Country Portfolio Evaluation (1994-2008), Global Environment Facility, Evaluation Office.

GHINET S. et DunAND C., 1998, «Les organisations locales face au marché. Leçons tirées de deux programmes de développement au Brésil et en Haïti », in DELER J.-P., PIVETEAU A. et ROCA P.-J., ONG et développement : société, économie et politique, Paris, Khartala, p. 381-397.

Hinnebusch Raymond et SCHMIDT Soren, 2008, The State and the Political Economy of Reform in

Syria, Boulder, CO, Lynne Rienner Publisher.

IRANI Khaled et JoHnson Chris, 1998, « Making it Pay: Can Community Based Biodiversity Conservation Programmes be Sustained through Market-Driven Income Schemes? », Paper Prepared for the International Workshop on Community-Based Natural Resource Management, Washington DC, May 10-14, 1998.

KARAM Karam, 2004, Revendiquer, mobiliser, participer: les associations civiles dans le Liban de l'après-guerre, Thèse de doctorat en Sciences politiques, Université Aix Marseille III. 
KARAM Karam, 2006, Le mouvement civil au Liban. Revendications, protestations et mobilisations associatives dans l'après-guerre, Paris, Karthala et Aix-en-Provence, IREMAM.

Kingston Paul, 2001, «Patrons, Clients and Civil Society: A Case Study Of Environmental Politics in Postwar Lebanon », Arab Studies Quarterly, Winter (version en ligne non paginée).

LE SAUX Mathieu, 2006, «Les dynamiques contradictoires du champ associatif syrien », REMMM, 115-116, p. 193-209.

MERRIEN François-Xavier, 1999, «La Nouvelle Gestion publique : un concept mythique », Lien social et Politiques, 41, p. 95-103.

Norton Augustus Richard (dir.), 1995, Civil Society in the Middle East, vol. 1, Leiden, Brill.

NorTon Augustus Richard (dir.), 1996, Civil Society in the Middle East, vol. 2, Leiden, Brill.

PerTHES Volker, 1997, The Political Economy of Syria under Asad, Londres, I.B.Tauris.

PNUD, s. d., Project Brief: Biodiversity Conservation and Protected Area Management (Syria),

Programme des Nations Unies pour le développement.

RUIZ DE ElVIRA Laura, 2010, «L'État syrien de Bachar Al-Assad à l'épreuve des ONG », Maghreb-Machrek, 203, p. 41-57.

SALAmON Lester, 1997, Holding the Center: America's Nonprofit Sector at a Crossroads, New York, Nathan Cummings Foundation.

SCHNEIDER Ingrid et BuRnetT G. Wesley, 2000, « Protected Area Management in Jordan », Environmental Management, 25-3, p. 241-246.

SERrA Gianluca, 2007, Ecotourism in the Palmyra Desert, Syria: A Feasibility Study, BirdLife International.

Smouts Marie-Claude, 2001, Forêts tropicales, jungle internationale. Les revers de l'écopolitique mondiale, Paris, Presses de Sciences Po.

UICN, 1994, La Protection environnementale en Islam, deuxième édition augmentée, Gland, UICN.

UICN et SNPL, 2007, Al Hima, A Way of Life, Amman, UICN et Beyrouth, SPNL.

WiKTOROWICZ Quintan, 2002, «The Political Limits to Nongovernmental Organizations in Jordan », World Development, 30-1, p. 77-92. 